\title{
Stochastic model for the Ros in a heterogeneous environment
}

\author{
$\underline{\text { Z. Jovanoski }}^{\text {a }}{ }^{\text {J. J. Sharples }}{ }^{\text {a, H. S. Sidhu }}{ }^{\text {a }}$ and I. N. Towers ${ }^{\text {a }}$

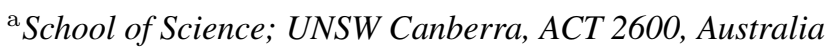 \\ Email: z.jovanoski@adfa.edu.au
}

\begin{abstract}
From a point source, landscape fires accelerate until they reach a quasi-equilibrium rate of spread. A fire is more easily suppressed by first-responders in its initial stages of development. As such, knowledge of the rate of acceleration of a fire from ignition can be valuable from a fire management perspective. However, the majority of studies in wildland fire science have been dedicated to development of models for the quasiequilibrium rate of spread, attained after its acceleration phase. Comparatively little attention has been given to the development of models that specifically account for the growth phase of a fire's development.

The rate of acceleration depends on many factors including variations in ambient and induced wind speed and direction, variation in moisture content of the fuel, fuel stratification and slope variation. Present models of fire growth from a point ignition are expressed as deterministic algebraic equations, thereby neglecting variability. The numerous variables involved make predictions of rate of spread from a point source very difficult.

We propose the use of stochastic differential equations to investigate the growth of a fire in both a homogeneous and heterogeneous environment. This approach provides a more realistic portrayal of the time series data of fire growth. It also allows for better discrimination of the mechanisms driving the growth phase of fire spread.
\end{abstract}

Keywords: Fire growth, fire acceleration, rate of spread, heterogeneous fuel, stochastic differential equation 


\section{INTRODUCTION}

Bushfires are a significant environmental problem. In the last decade this has become increasingly apparent, with several major conflagrations causing extensive loss of life and property, and considerable environmental damage. Regardless of the ultimate size and intensity of a bushfire, all fires start small - often originating as a point ignition, and growing in a manner that is governed by the ambient environmental conditions. Under certain conditions a bushfire will deposit firebrands ahead of the main front, igniting spot-fires, which can also be viewed as evolving from a point source.

Landscape fires originating from a point source accelerate until they reach a quasi-equilibrium rate of spread. This behaviour has been confirmed in laboratory experiments (McAlpine and Wakimoto, 1991). Attacking fires while they are in their acceleration phase, before they have attained their potential quasi-equilibrium rate of spread, provides a greater chance of suppression success (Cheney and Gould, 1997). As such, there are good reasons to understand the likely behaviour of a fire during its acceleration phase, and mathematical modelling provides a means to improve this understanding. However, in comparison with the vast literature that deals with modelling the quasi-equilibrium rate of spread, there is little that specifically considers this acceleration phase of a fire's development (Cruz et al., 2015). The way a fire develops during its acceleration phase depends on the local environmental conditions, including variations in ambient and induced wind speed and direction, and variations in moisture content and structure of the fuel. Even when a fire attains quasi-equilibrium state, its rate of spread can still exhibit considerable variation about a mean value.

However, present models of fire growth from ignition are expressed as deterministic algebraic equations, thereby neglecting variability. Parameters in such models are estimated using nonlinear regression of empirical data, and once determined they are considered as fixed; separate estimates are required for different fuel types and environmental conditions. Any resulting model lacks flexibility and should not be used in circumstances where the fuel and environmental conditions are heterogeneous. Moreover, interactions between winds, terrain, different vegetation types and structures means that the conditions affecting the initial growth of a fire (and its longer-term propagation) can never be adequately quantified in solely deterministic terms. Coupling between the fire and the atmosphere further confound this situation. The intrinsic variability of the factors affecting the initial growth of a fire means that deterministic modelling approaches are ill-suited to modelling this aspect of fire spread. Indeed, such approaches gloss over the detailed dynamics, which are inherently stochastic in nature due to the turbulent conditions under which fires develop. Recent advances in stochastic dynamical systems theory and their applications, as well as in our understanding of dynamic fire behaviour, means that we are now able to explicitly address the acceleration phase from a point ignition.

The models we propose consider the acceleration phase of a fires growth as a stochastic dynamical system. Such an approach permits a probabilistic interpretation of fire growth, which allows for estimation of worstcase and mean-case scenarios. Also, by explicitly accounting for the intrinsic variability of fire spread and the environmental conditions that drive it, our stochastic approach has the potential to provide greater insights into the physical processes governing fire growth.

\section{MATHEMATICAL MODELLING OF THE RATE OF SPREAD}

The rate of spread (Ros) data used in this study are taken from McAlpine and Wakimoto (1991) and is reproduced in Figure 1. It is derived from distance-time measurements of wind tunnel experiments (McAlpine 1988). The data set represents five replicates and for each the fuel load was $26.3 \mathrm{~kg} / \mathrm{m}^{3}$ and burned under the influence of a wind speed of $4.8 \mathrm{~km} / \mathrm{hour}$. The temperature $\left(26.7^{\circ} \mathrm{C}\right)$ and humidity $(80 \%)$ were controlled for. The fuel moisture content was measured just prior to burning, and the mean of the five replicates was $8.62 \%$ (standard deviation of 0.57 ). The variability in the data may be due to differing fuel moisture content, variability in the packing of the fuel bed for each experimental run (Finney, 2004), and turbulence induced by the fire itself. We treat each of the replicates in Figure 1 as independent data sets.

\subsection{Curve-fitting: Homogeneous environment}

Following Viegas (2004) the position $x(t)$ of the fire front at a given time $t$ since ignition is given by

$$
x(t)=\int_{0}^{t} R(s) d s,
$$

where $R(t)$ is the Ros. If $R(t)$ is a constant, the fire propagates at a constant speed $R_{e}$ say, then $x(t)=R_{e} t$, as one would expect. In real fires however, one cannot expect the Ros to remain constant with time. With regard 
Z. Jovanoski, J. J. Sharples et al., Stochastic model for the RoS in a heterogeneous environment

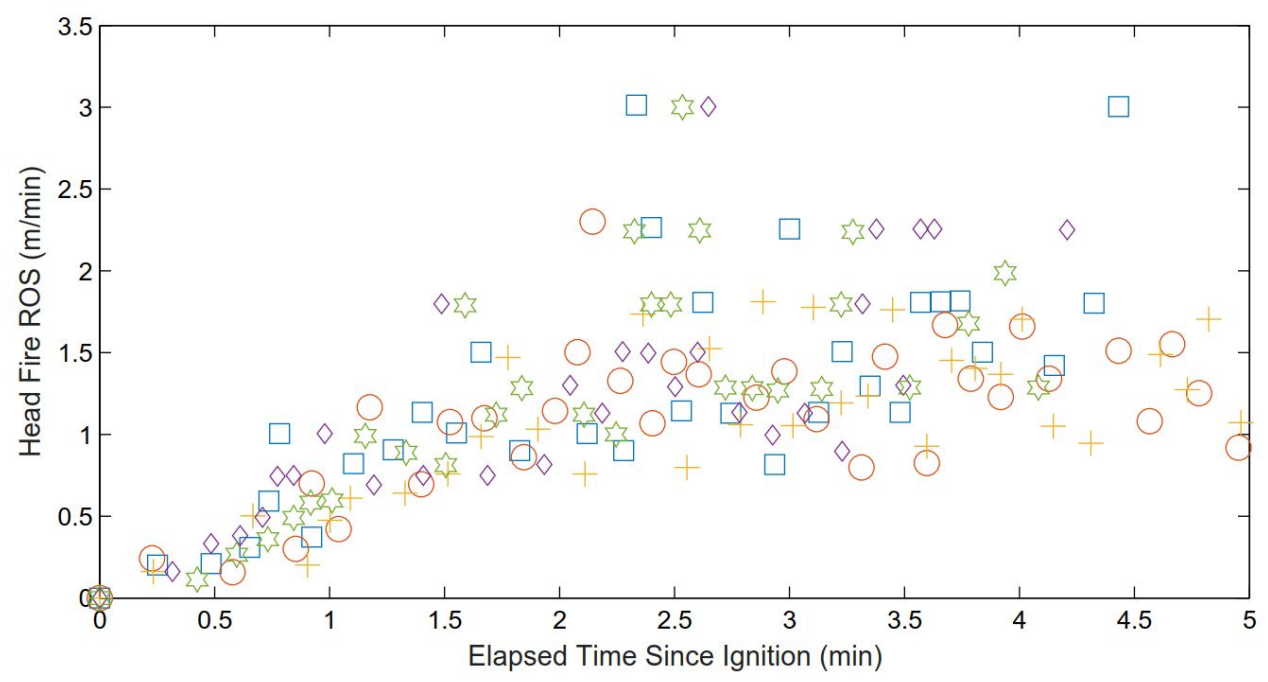

Figure 1. Observed rate of spread for a needle fuel bed, with a bulk density of $26.3 \mathrm{~kg} / \mathrm{m}^{3}$ and burned at a wind speed of $4.8 \mathrm{~km} / \mathrm{hr}$. Five replications are shown, each symbol (square, circle, etc.) being a separate replication. (Figure recreated from McAlpine and Wakimoto (1991).)

to the data in Figure 1, it appears there is an initial rapid increase in the Ros until reaching an inflection point, where the acceleration is at maximum. Beyond this point, the acceleration starts to decrease, asymptotically approaching the quasi-equilibrium Ros. To describe this behaviour, we propose a sigmoid (logistic) function for the Ros,

$$
R(t)=\frac{R_{0} R_{e}}{R_{0}+\left(R_{e}-R_{0}\right) e^{-a t}} .
$$

Here $R_{0}$ is the initial value of the Ros, the quasi-equilibrium value is $R_{e}$ and $a$ is the rate of increase of the Ros. Then according to (1) the distance travelled by the fire front is

$$
x(t)=\frac{R_{e}}{a} \ln \left(R_{e}-R_{0}+R_{0} e^{a t}\right)-\frac{R_{e}}{a} \ln \left(R_{e}\right) .
$$

Following Sanni et al. (2013), the aim is to estimate the three parameters, $R_{0}, R_{e}$ and $a$, by implementing a nonlinear least-squares method to minimise the sum of the square of the errors between the observed and the curve-fitted values.

For illustrative purposes we fitted (2) to a single replicate of the distance-time data and (3) to its associated Ros data (the squares), respectively. The Levenberg-Marquardt algorithm was used to solve the nonlinearleast squares problem (Rowlings et al., 1998). The estimated parameters including a 95\% confidence interval (CI) is shown in Table 1. For the distance data, all three of the estimated parameters are statistically significant (at the $p=0.05$ significance level). The Root-Mean-Squared-Error (RMSE) is close to zero and both $\mathrm{R}^{2}$ and ADJ $\mathrm{R}^{2}$ are approximately one, all indicating a very good fit to the data.

Initial attempts to estimate the parameters based on the Ros data resulted in statistically significant estimates for both $R_{e}$ and $a$ but not for $R_{0}$. This is due to the large variability in the Ros data, a quantity derived from the distance-time data. Therefore, to make progress, we set $R_{0}=0.170$ and estimated $R_{e}$ and $a$. Table 1 shows the two estimated parameters differ slightly between the distance and Ros data. There is an order of magnitude difference in the RMSE resulting in wider $95 \%$ CIs for both parameters. The $\mathrm{R}^{2}$ and ADJ $\mathrm{R}^{2}$ indicate that model (2) explains roughly $66 \%$ of the variability in the data.

\subsection{An SDE approach: Heterogeneous environment}

An alternative to nonlinear regression is to reformulate the model as a differential equation, specifically as a stochastic differential equation (SDE). Using an SDE allows for the inclusion of local environmental variability that might be due to heterogeneous fuels or changes in wind direction and strength. 
Z. Jovanoski, J. J. Sharples et al., Stochastic model for the RoS in a heterogeneous environment

Table 1. Nonlinear curve-fitting to a single replicate (square symbol).

\begin{tabular}{|c|c|c|c|c|c|c|}
\hline Parameter & $R_{0}$ & $R_{e}$ & $a$ & RMSE & $\mathrm{R}^{2}$ & ADJ $R^{2}$ \\
\hline Distance & 0.170 & 1.416 & 1.927 & 0.051 & 0.999 & 0.999 \\
\hline $95 \%$ CI & $(0.121,0.219)$ & $(1.366,1.466)$ & $(1.567,2.288)$ & & & \\
\hline Ros & 0.170 & 1.550 & 1.827 & 0.426 & 0.665 & 0.654 \\
\hline $95 \%$ CI & & $(1.305,1.809)$ & $(1.165,2.485)$ & & & \\
\hline
\end{tabular}

With reference to Figure 1, during the early development of the fire, $R^{\prime}(t) \propto R(t)$, then as $R(t) \rightarrow$ $R_{e}, R^{\prime}(t) \rightarrow 0$. This behaviour is captured by the logistic differential equation,

$$
\frac{d R}{d t}=a R\left(1-\frac{R}{R_{e}}\right)
$$

It is worth noting that equation (4) is related to the power-law nonlinear model of Dold and Zionviev (2009), for the modelling of fire eruptions. Under certain conditions the rate of change of the Ros satisfies

$$
\frac{d R}{d t}=a R\left(1-\left(\frac{R}{R_{e}}\right)^{\frac{1}{\nu}-1}\right),
$$

with $\nu<1$. Setting $\nu=1 / 2$, we arrive at equation (4). For this model, as with the equation (4), there exist two equilibrium points, namely $R(t)=0$ (unstable) and $R(t)=R_{e}$ (stable). This means any slight perturbation off the $R(t)=0$ equilibrium state, the Ros will accelerate towards the $R(t)=R_{e}$ state.

In what follows is a preliminary attempt to model the fire's growth from ignition using an SDE. The manner in which stochasticity is added is a modelling issue, we therefore offer two approaches: The first is based on the variability in the rate of change of the Ros, while the second approach treats the parameters, specifically the 'local' quasi-equilibrium as a stochastic state-variable.

Variable growth rate. The stochastic version of the logistic differential equation written in the Ito form is (Gardiner, 2009),

$$
d R=a R\left(1-\frac{R}{R_{e}}\right) d t+\sigma R d W, \quad R(0)=R_{0},
$$

where the parameters $R_{0}, R_{e}$ and $a$ have the same meaning as before. The parameter $\sigma$ is the strength of the local environmental variability and $W(t)$ is a standard Wiener process with expected value $\mathbb{E}[W]=0$ and variance $\mathbb{V}[W(t)]=t$. Equation (6) incorporates stochasticity by aggregating the effect of growth and self-limiting of the fire spread, both of which are affected by local environmental variability, into a single term. The appearance of $R(t)$ in the stochastic term implies that the variance increases with increase in the Ros, which is consistent with the data.

The solution to (6) is accomplished via the Euler-Maruyama algorithm (Higham. 2001). The numerical solution to the SDE is a single realisation, what is of importance is to determine the expected value and variance of a large number of such realisations. To solve equation (6) we must specify four parameters: $R_{0}, R_{e}, a$ and $\sigma$. Strictly speaking we should estimate these parameters by using, for example, a maximum-likelihood method applied to (6) (Kloeden et al., 1994). Here, however, we make use of the values already determined and our tasks remains to find $\sigma$. This is done by searching through a range of values of $\sigma$ for which the estimated variance $\mathbb{V}[R(t)]$ (after quasi-equilibrium is reached) is equal to the RMSE. This is justifiable if we seek to make a comparison between (2) and (6) (Sanni et al., 2013).

To estimate both $\mathbb{E}[R(t)]$ and variance $\mathbb{V}[R(t)]$ we employ a Monte-Carlo method to generate 100,000 realisations. Figure 2 shows the expected Ros with a 95\% prediction interval (PI). Requiring $\mathbb{V}[R(t)] \approx$ RMSE yields $\sigma=0.545$. It is clear that almost all of the data resides within the $95 \%$ PI, suggesting that the proposed model provides a good description of the observed Ros. 
Z. Jovanoski, J. J. Sharples et al., Stochastic model for the RoS in a heterogeneous environment

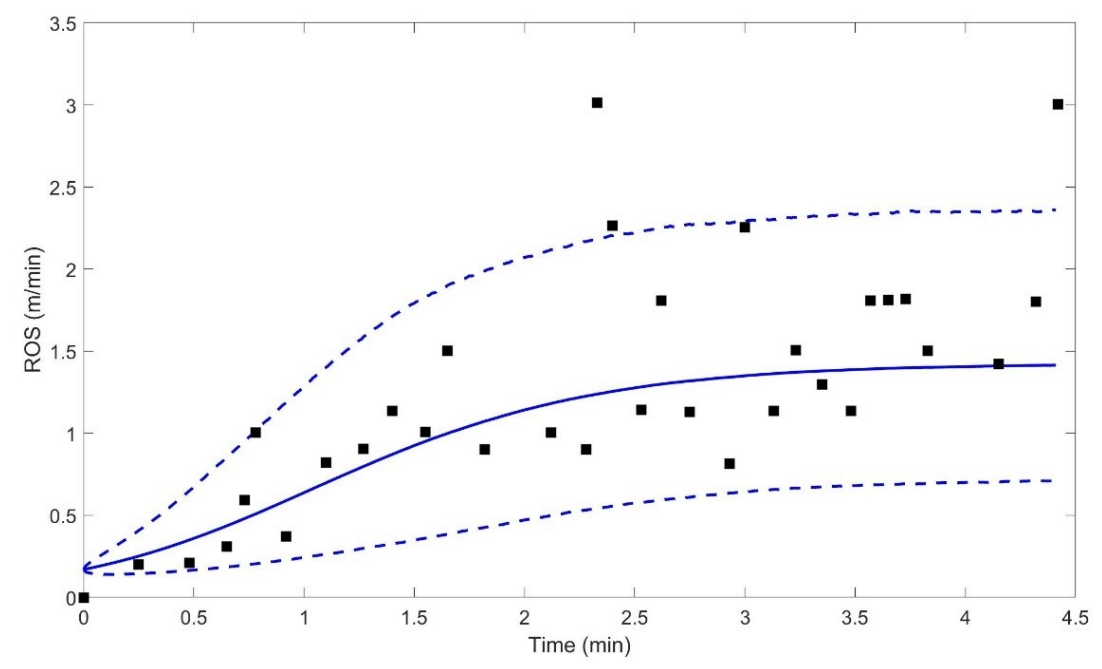

Figure 2. Plot of the expected Ros based on 100,000 realisations of the SDE. Also shown is the $95 \%$ prediction interval for a new realisation of the SDE.

The lower of the 95\% PI offers practical information about the likelihood of the fire self-extinguishing, often referred to as a 'go/no-go' threshold. The closer the lower prediction interval is to zero, the more likely the fire will self-extinguish. The upper prediction interval provides important information for fire management, as it relates to the potential for rapid initial growth of a fire over the landscape. Knowledge of the upper prediction interval can therefore give fire managers a better idea of the operational window for initial response once an ignition occurs, thus enabling more informed deployment of personnel and assets to head off a fire. Sanni et al. (2013) provides a comparison of the SDE with the nonlinear regression. The main finding is that for fire whose behaviour is more in accord with the SDE model is less likely to self-extinguish, and has the potential to exhibit higher rates of spread.

Variable $\boldsymbol{R}_{e}$. Making predictions based on nonlinear regression requires that the parameters be determined and kept fixed for a given set of environmental conditions. This means a change in wind speed or fuel type requires equation (2) be refitted. This is also true of (6): the model is inadequate as it cannot discriminate how different conditions can impact fire growth and acceleration. This limits the usefulness and application of both approaches.

Another way to incorporate stochasticity is by treating some of the parameters as state-variables. Suppose the 'local' quasi-equilibrium rate of spread $R_{e}(t)$ is now a state-variable and is a function of the wind velocity. We consider the effect of changes in wind speed and direction. The simplest of such models is described by a stochastic process

$$
\begin{aligned}
\frac{d R}{d t} & =a R\left(1-\frac{R}{R_{e}}\right), \\
d R_{e} & =\sigma d W .
\end{aligned}
$$

Here $d W(t)$ represents a change in the local wind direction and strength; $d W(t)>0$ helps to increase $R_{e}$, while $d W(t)<0$ to decrease $R_{e}$. The exact solution is $R_{e}(t)=R_{e}(0)+\sigma W(t)$ with $\mathbb{E}\left[R_{e}(t)\right]=R_{e}(0)$ and $\mathbb{V}\left[R_{e}(t)\right]=\sigma^{2} t$.

Figure 3 depicts three realisations with $R_{e}(0)=1.550$. One realisation (blue) is the effect of a number of favourable wind changes that have increased the local $R_{e}(t)$ thus increasing $R(t)$. Due to a series of unfavourable wind changes in another realisation (green) the $R_{e}(t)$ is observed to reach zero, this being interpreted as the fire having self-extinguished. We are now in a position to estimate the probability that a fire may self-extinguish. Table 2 summarises the results based on 10,000 realisations of equations (7) and (8) for different levels of noise intensity. The probability increases as the ratio $R_{e}(0) / \sigma$ decreases. 


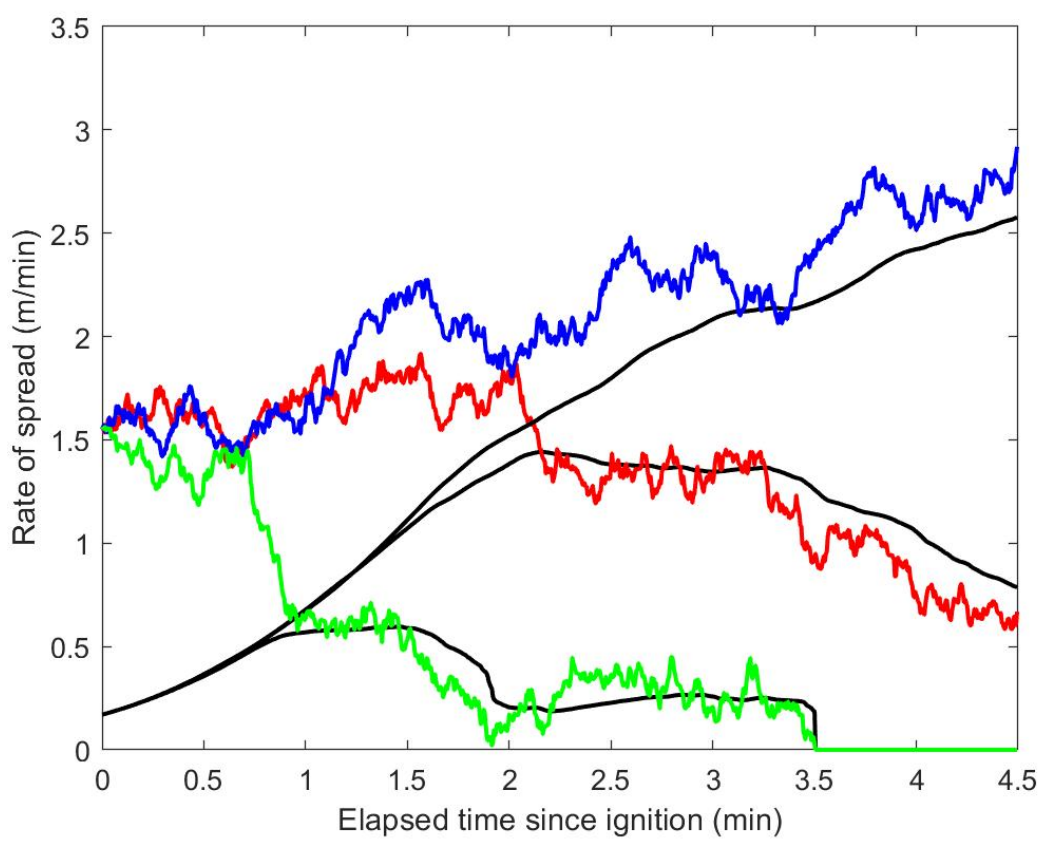

Figure 3. Plot of three different realisations of the local quasi-equilibrium and the solution (black) for the Ros based on equations (7) and (8). One realisation is seen to self-extinguish (green).

The idea that the Ros depends on the energetic ratio balance between the heat released by combustion and the energy necessary for fuel ignition is well known (Viegas, 2004). Certainly the energy required for ignition of moist fuel is greater than that of dry fuel, thus moist fuel will have a dampening effect on $R_{e}$. Based on experiments, Rossa (2017) developed an empirical model for $R_{e}$ that is a power-law of the moisture content of the fuel. This could be incorporated into equations (7) and (8) to model the effect of a fuel with variable moisture content on the Ros. Such models are currently being developed.

Table 2. Estimate of the probability to self-extinguish based on 10,000 realisations of equations (7) and (8).

\begin{tabular}{|c||cccc|}
\hline $\boldsymbol{\sigma}$ & 0.2 & 0.4 & 0.6 & 0.8 \\
\hline Probability & $2.6 \times 10^{-4}$ & $6.8 \times 10^{-2}$ & 0.22 & 0.36 \\
\hline
\end{tabular}

Lastly, another important effect on the Ros is the curvature of the fire front. For a point-source ignition, the fire front curvature is large and as the fuel burns the radius of the fire front increases, the curvature decreases, until at such time the expanding ring of fire essentially behaves as a line fire. Point-source ignition takes a longer time than a line ignition to accelerate to $R_{e}$ (McRae, 1999). However, differences can be variable and depend on the types of fuels (Finney and McAllister, 2001). Weber (1989) developed a theoretical framework for the Ros from a point-source ignition, and the conjoining of this model with that of equations (7) and (8) may be used to model the evolution of a point-source ignition to a line fire subject to variable environmental conditions.

\section{CONCLUSIONS}

In developing models for the spread of fire it is customary to fit the data based on some prescribed functional form. Such methods provide good descriptions of the time evolution of the mean Ros from time of ignition. The aim is to obtain parameter estimates and relevant CIs of the fixed parameters for the spread rate. Alternatively, an SDE formulation is a natural way to describe the rate of change of the Ros. Carrying out a large 
number of simulations enables the construction of an empirical probability density function for the Ros, from which statistical quantities can be determined. This has practical implications for the management of fires.

The model we propose explicitly incorporates the stochastic dynamics of fire spread and environmental variability. The model is appropriate for assessing the likely development of fires in the landscape. The model establishes a probabilistic framework that acknowledges the uncertainties inherent in fire spread.

The SDE formulation for the spread rate is particularly attractive as it provides a way to explicitly involve a fire's history (Viegas, 2004). This could be accomplished by using either a distributed time-delay in the spread rate, which involves a weighted average of the spread rate taken over all previous times, or as a discrete timedelay due to the process of pyrogenesis (Dold and Zionviev, 2009). This line of research is currently being investigated.

\section{REFERENCES}

Cheney, N. P. and J. S. Gould (1997). Fire growth and acceleration. International Journal of Wildland Fire 7 , $1-5$.

Cruz, M. G., J. S. Gould, M. E. Alexander, A. L. Sullivan, W. L. McCaw, and S. Matthews (2015). A guide to rate of fire spread models for australian vegetation. Technical report, CSIRO Land and Water Flagship, Canberra, ACT, and AFAC, Melbourne, Vic, 123pp.

Dold, J. W. and A. Zionviev (2009). Fire eruption through intensity and spread-rate interaction mediated by flow attachment. Combustion Theory and Modelling 13, 763-793.

Finney, M. A. (2004). Farsite: Fire area simulator - model development and evaluation. Technical report, USDA For. Serv., Rocky Mt. Res. Stn., Ogden, UT. Res. Pap. RMRS-RP-4 Revised. 47 p.

Finney, M. A. and S. S. McAllister (2001). A review of fire interactions and mass fires. Journal of Combustion 2011, article ID 548328 (14 pages).

Gardiner, C. (2009). Stochastic Methods: A handbook for the natural and social sciences. Berlin: SpringerVerlag.

Higham, D. J. (2001). An algorithmic introduction to numerical simulation of stochastic differential equations. SIAM. Review 43, 525-546.

Kloeden, P. E., E. Platen, and H. Schurz (1994). Numerical solution of SDE through computer experiments. Berlin: Springer-Verlag.

McAlpine, R. S. (1988). Acceleration of point source fire to equilibrium speed. In Theses, Dissertations, Professional Papers. Paper 1465.

McAlpine, R. S. and R. H. Wakimoto (1991). The acceleration of fire from point source to equilibrium spread. Forest Science 37, 1314-1337.

McRae, D. J. (1999). Point-source fire growth in jack pine slash. International Journal of Wildland Fire 9 , 65-77.

Rossa, C. G. (2017). The effect of fuel moisture content on the spread rate of forest fires in the absence of wind or slope. International Journal of Wildland Fire 26, 24-31.

Rowlings, J. O., S. G. Pantula, and D. A. Dickey (1998). Applied regression analysis: a research tool (2 ed.). New York: Springer-Verlag.

Sanni, S., J. J. Sharples, A. M. Gill, Z. Jovanoski, H. S. Sidhu, and I. N. Towers (2013). A stochastic differential equation approach to modelling the growth phase of fire spread. In In Proceedings of MODSIM2017, 22nd International Congress on Modelling and Simulation (pp. 1152-1148). Hobart, Australia.

Viegas, D. X. (2004). On the existence of a steady state regime for slope and wind driven fires. International Journal of Wildland Fire 13, 101-117.

Weber, R. O. (1989). Analytic models for fire spread due to radiation. Combustion and Flame 78, 398-408. 